\title{
An invention of baton dance? exercise regime on obesity diagnosis among sedentary adults
}

\begin{abstract}
The purpose of this investigation is to evaluate the effect of a 12-weeks of Baton Dance ${ }^{\mathrm{TM}}$ exercise, an invention in the treatment of obesity diagnosis among overweight sedentary adults in higher learning organizations. Baton Dance ${ }^{\mathrm{TM}}$ exercise program is a combination of aerobic dance activity, circuit training, and interval training which improvise own body weight during the exercise program. It combines physical training that includes alternating of low and medium intensity which involves a physical conditioning training with medium volume and low resistant with a short rest time. Forty subjects between the ages of 25 to 55 years, have had a diagnosis as sedentary, were overweight and have a fitness score below the mean. Subjects were randomly assigned to a 12-week of either Baton Dance $^{\mathrm{TM}}$ as a treatment group or an aerobics dance exercise as the control group. A pre-testpost-test control research design was utilized. Both groups met for 50 minutes, three times a week for a total of 36 sessions. Statistical analysis includes (group $x$ time) paired t-test and independent sample t-test were used to determine between and within group mean differences. Results suggested that subjects in the Baton Dance ${ }^{\mathrm{TM}}$ exercise regime intervention experienced positive improvement in obesity diagnosis after 12 weeks of treatment within the group effect $\mathrm{p}<.05$. Results for obesity diagnosis were, BMI (mean pre: 30.57, mean post: 29.24), PBF from (mean pre: 43.52, mean post: 43.00), WHR (mean pre: .928, mean post: .924), SMM (mean pre: 23.23, mean post: 23.32). Therefore, it was concluded that the intervention of Baton Dance ${ }^{\mathrm{TM}}$ exercise program had improved obesity diagnosis, and contributed to a positive findings among overweight sedentary adults in higher education organization.
\end{abstract}

Keyword: Baton dance ${ }^{\mathrm{TM}}$; Circuit training; Interval training; Aerobics; Dance; Body mass index (BMI); Percent body fat (PBF); Skeletal muscle mass (SMM); Obesity; Sedentar 